CASE REPORT

\author{
A.K. Kim \\ E.B. Lee \\ L.J. Bagley \\ L.A. Loevner
}

\section{Retained Surgical Sponges after Craniotomies: Imaging Appearances and Complications}

SUMMARY: Imaging within 24 to 48 hours after most neurosurgical procedures is a routine practice. Nonresorbable surgical sponges have radiopaque filaments readily visible on CT scans and plain film radiographs. However, the proton-poor barium sulfate responsible for this radio-opacity is generally not detectable on MR imaging in the immediate post-operative period. Findings on MR imaging become more evident with elapsing time and when a foreign-body reaction to the sponge manifests as a mass lesion, which can mimic residual or recurrent intracranial tumor or abscess. Although preventive measures by our surgical colleagues to ensure accurate and correct sponge counts before and after wound closure is paramount, even the most fastidious efforts may rarely result in an inadvertently retained surgical sponge. The role of the radiologist is to recognize the imaging findings of this entity and its potential complications so that appropriate and prompt management can be initiated.
$\mathbf{R}^{\mathrm{e}}$ etained surgical materials and their potential adverse outcomes are infrequent but important complications that have been well described after abdominal and pelvic surgery. ${ }^{1-2}$ In contrast, a limited number of studies have been reported regarding retained surgical sponges after craniotomies. It is important that the radiologist be aware of this entity; its imaging appearance; and its potential complications, which include the formation of granulomas and abscesses. These complications may be mistaken for residual or recurrent neoplasms. We discuss the imaging appearance of retained surgical sponges after neurosurgical procedures in 3 patients and review the potential complications should these go unidentified.

\section{Case Reports}

\section{Case 1}

A 44-year-old man with no significant medical history was admitted from the emergency department with a 3-week history of fever; headache; and progressive cognitive decline, including memory loss and confusion. Initial brain MR imaging showed increased T2-weighted fluid-attenuated inversion recovery signal intensity in the left hippocampus with regions of leptomeningeal enhancement. Differential considerations included herpes encephalitis, limbic encephalitis, or infiltrating glioma. The patient was empirically treated with acyclovir for possible herpes encephalitis, with no improvement in his clinical condition. Analysis of CSF and hematologic evaluation were negative for malignant or infectious sources. The unclear cause of the patient's symptoms prompted a brain biopsy, results of which revealed acute hemorrhagic leukoencephalopathy. Routine postoperative head CT scan performed within 12 hours of surgery showed a serpentine hyperattenuated object in the surgical bed suspicious for a retained surgical sponge (Fig 1). Surgical exploration confirmed a retained cottonoid sponge that was removed.

Received November 17, 2008; accepted November 19.

From the Departments of Neuroradiology (A.K., L.J.B., L.A.L.) and Pathology (E.B.L.), University of Pennsylvania, Philadelphia, $\mathrm{Pa}$

Previously presented as a poster at: Annual Meeting of the American Society of Neuroradiology, June 2-5, 2008, New Orleans, La.

Please address correspondence to Laurie A. Loevner, MD, Department of Neuroradiology, University of Pennsylvania Health System, 3400 Spruce St, 2 Dullas Bldg, Philadelphia, PA 19104; e-mail: laurie.loevner@uphs.upenn.edu

DOl 10.3174/ajnr.A1469

\section{Case 2}

A 52-year-old woman underwent a right pterional craniotomy for resection of a right frontal atypical meningioma. Routine postoperative CT scan performed within 12 hours of surgery revealed multiple curvilinear hyperattenuated structures in the right infratemporal fossa, thought to represent surgical clips (Fig 2). On routine postoperative MR imaging performed within 12 hours of surgery, these curvilinear structures in the right infratemporal fossa surgical bed were not identified because they were obscured by susceptibility artifacts caused by blood products and air. Routine follow-up brain MR imaging study performed at an outside institution 4 months after the initial surgery showed a nodular enhancing "mass" in the right infratemporal fossa, which was interpreted as concerning for recurrent meningioma. The patient underwent a second craniotomy. On intraoperative inspection, the mass was found encased within the right temporalis muscle and, on gross inspection, had an attenuated fibrous capsule with apparent tufts of cottonoid material embedded within it. Gross pathologic evaluation demonstrated several portions of blue plastic foreign material (surgical sponge) present within a rubbery, tan-pink, soft tissue mass. On microscopic inspection, foreign material with suture admixed in a giant-cell granulomatous response was identified. No neoplasm was present.

\section{Case 3}

A 65-year-old man was admitted from the trauma bay after sustaining a major head traumatic injury from an assault. Initial CT examination of the head showed multiple calvarial fractures, extensive intracranial hemorrhage, and edema with mass effect. Increasing parenchymal edema and mass effect on serial CT studies in combination with declining responsiveness prompted decompressive right hemispheric craniectomy 5 days after admission. During the operation, there was considerable bleeding from the superior sagittal sinus that was controlled with multiple cottonoid sponges. Before wound closure, all but 1 of the cottonoid sponges could be safely removed. Routine postoperative head CT scan performed within 12 hours of the surgery revealed a serpentine hyperattenuated retained surgical sponge adjacent to the superior sagittal sinus at the vertex (Fig $3 A$ ). MR imaging examination showed the surgical sponge as a curvilinear T2-weighted hypointense structure (Fig $3 B$ ) that demonstrated susceptibility artifacts on the gradient-echo sequence. The sponge could not be removed, and the patient was eventually discharged to a rehabilitation center. 

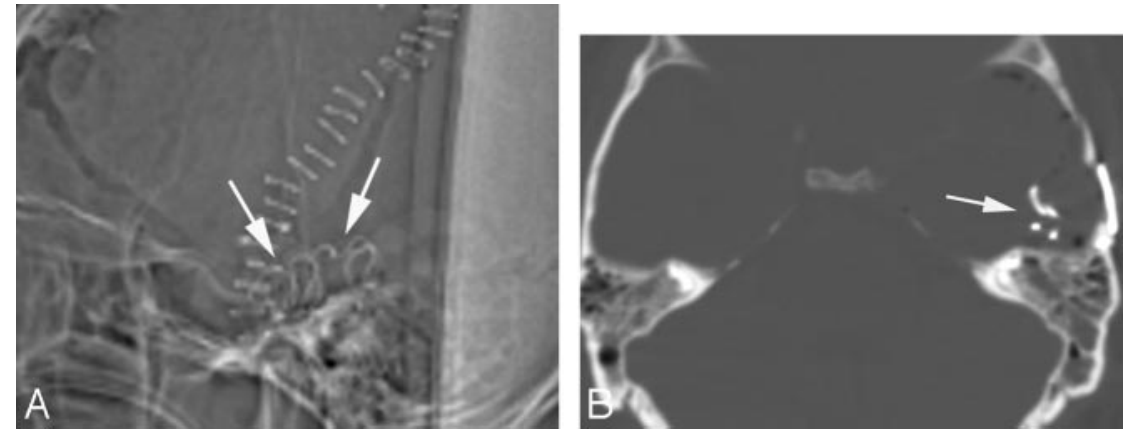

Fig 1. $A$, Sagittal topogram from unenhanced $C T$ scan shows a hyperattenuated curvilinear object overlying the surgical field, representing a retained surgical sponge. $B$, Axial $C T$ scan in bone window shows the hyperattenuated serpiginous barium-impregnated filaments of the retained cottonoid sponge in the left middle cranial fossa.
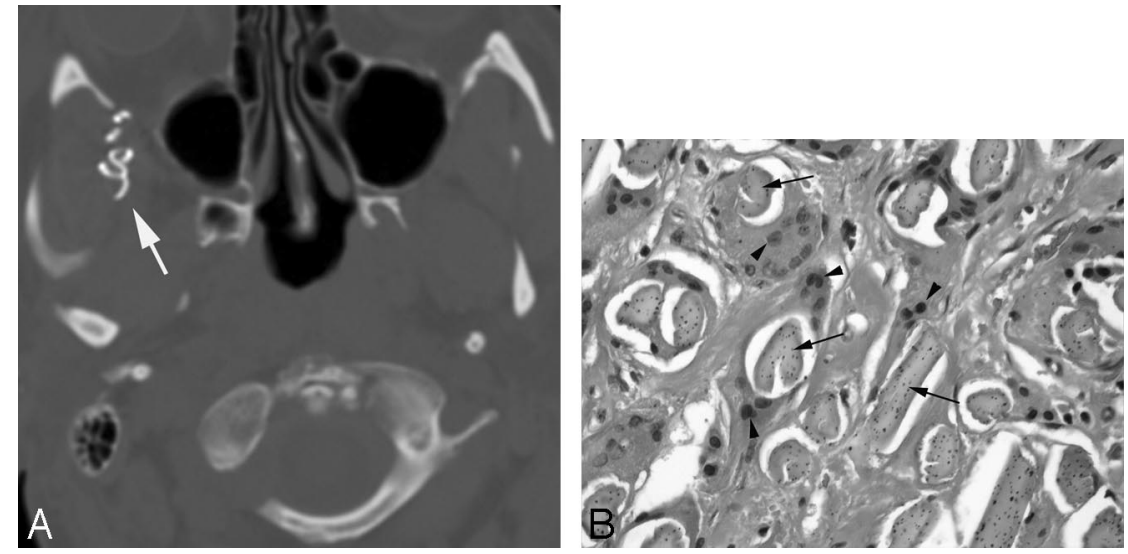

Fig 2. A, Axial CT scan in bone window at the level of the infratemporal fossa shows a hyperattenuated serpiginous structure (arrow) in the right infratemporal fossa compatible with a retained surgical sponge. $B$, Microphotograph at high power demonstrates multinucleated foreign-body giant cells (arrowheads) engulfing foreign-body material (arrows; hematoxylin-eosin, original magnification $\times 40$ ).
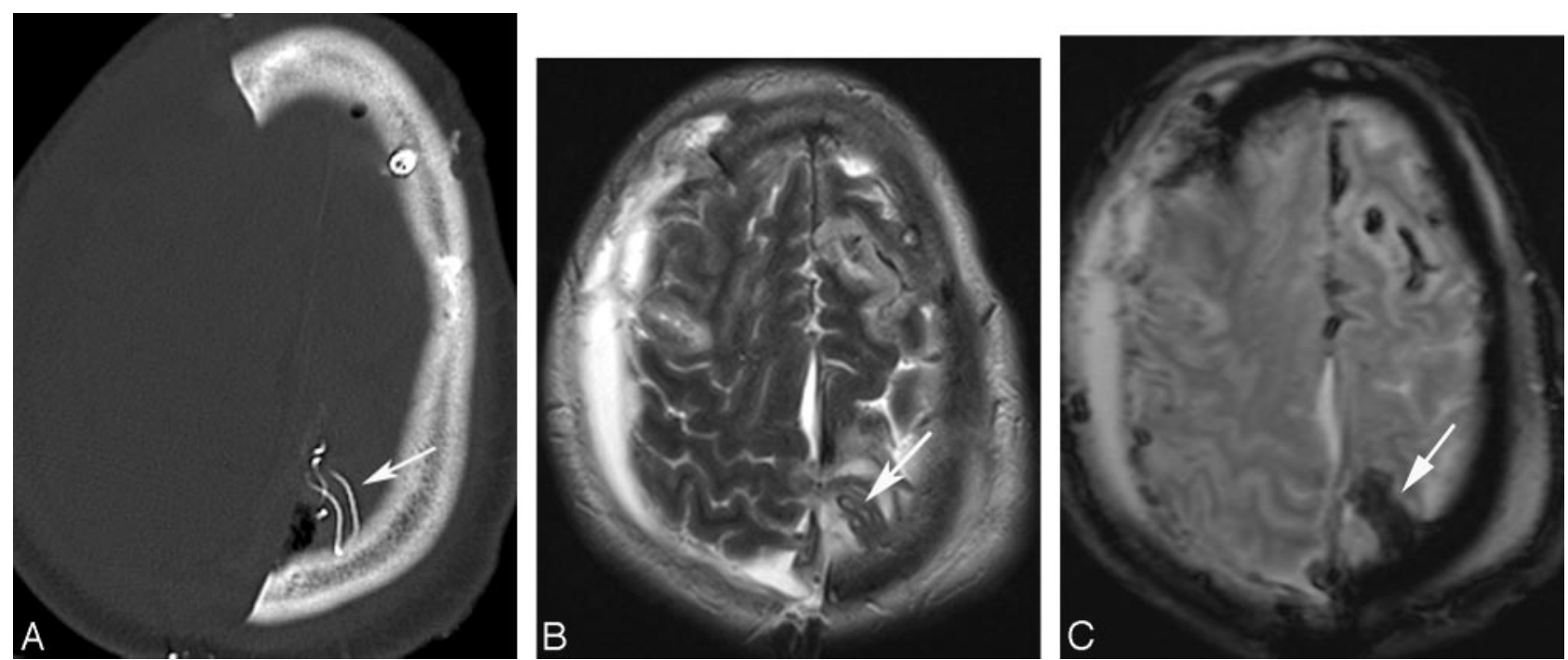

Fig 3. A, Axial unenhanced CT study shows the characteristic hyperattenuated serpentine object (arrow) representing the barium-impregnated filaments of a retained cottonoid sponge $B$, Axial T2-weighted image shows the retained surgical sponge (arrow) as a hypointense serpentine foreign body that shows susceptibility effects (arrow) on the gradient-echo sequence $(C)$.

\section{Discussion}

Gossypiboma is a term used to describe a foreign-body reaction surrounding material made of cotton matrix. The term is derived from the Latin word gossypium, meaning "cotton" and the Swahili term boma, meaning "place of concealment" and historically has been used, along with the term textiloma, to describe the foreign-body granulomatous reactions that occur in response to inadvertently retained surgical sponges. Other less commonly used terms are gauzoma (from surgical gauze) and muslinoma (from muslin, a woven cotton fabric).

Two types of foreign-body reactions generally occur in response to a retained surgical sponge. There can be an aseptic 
fibrous reaction that involves adhesion formation, encapsulation, and granulomatous pseudotumoral formation as in the patient in case 2. Alternatively, there can be an exudative reaction resulting in abscess formation. Although exudative responses typically manifest in the early postoperative stage, granulomatous responses can remain clinically silent for years. ${ }^{3}$

Neurosurgeons and other surgical subspecialists routinely use hemostatic resorbable and nonresorbable agents. Bioabsorbable agents may be left in the surgical bed to maintain hemostasis after wound closure. Three commonly used resorbable hemostatic agents are Gelfoam gelatin foam (Pfizer, New York, NY), Surgicel oxidized cellulose (Ethicon Endo-Surgery, Cincinnati, Ohio), and Avitene microfibrillar collagen (Davol, Cranston, RI). ${ }^{4}$ Nonresorbable agents are removed before wound closure. An exception to the nonresorbable agents is muslin, which is a cotton fabric occasionally used to reinforce aneurysmal walls during surgical repair of intracranial aneurysms. Although there are case reports describing an excessive inflammatory response to resorbable hemostatic agents ${ }^{5}$ and muslin, ${ }^{6}$ this is more commonly observed with the nonresorbable materials. Nonresorbable hemostatic agents commonly used in neurosurgical procedures are synthetic rayon fibers fashioned into strips or pledgets (cottonoids and kites) containing filaments impregnated with radiopaque barium sulfate that allow visualization on plain radiographs or on CT scans. ${ }^{4}$

Imaging within 24 to 48 hours after most neurosurgical procedures is routine practice. Whereas resorbable hemostatic agents may be difficult to appreciate on CT, especially in the early postoperative phase, nonresorbable surgical sponges have radiopaque filaments readily visible on CT scans and on plain radiographs. These filaments appear as curvilinear hyperattenuated structures. However, the proton-poor barium sulfate responsible for this radio-opacity is generally not de- tectable on MR imaging. The MR appearance of retained foreign-body material will depend on the time elapsed since surgery, the type of foreign-body reaction that occurs, and on the substance of the foreign material itself. Imaging findings on MR imaging become more evident when the foreign-body reaction manifests as a mass lesion, which can mimic residual or recurrent intracranial tumor or abscess. ${ }^{4,7}$

Cotton and gauze pads used in the surgical field should always have a tag that allows them to be easily located and removed, and all materials that are placed within the wound temporarily must be counted with meticulous care. ${ }^{8}$ Although preventive measures by our surgical colleagues to ensure accurate and correct sponge counts before and after wound closure is paramount, even the most fastidious efforts may result in an inadvertently retained surgical sponge. The role of the radiologist is to recognize the imaging findings of this entity and its potential complications so that appropriate and prompt management can be initiated.

\section{References}

1. O'Connor AR, Coakley FD, Meng MV, et al. Imaging of retained surgica sponges in the abdomen and pelvis. AJR Am J Roentgenol 2003;180:481-89

2. Gawande AA, Studdert DM, Orav EJ, et al. Risk factors for retained instruments and sponges after surgery. N Engl J Med 2003;348:229-35

3. Sakayama K, Fujibuchi T, Sugawara Y, et al. A 40-year-old gossypiboma (foreign body granuloma) mimicking a malignant femoral surface tumor. Skel Radiol 2005;34:221-24

4. Ribalta T, McCutcheon IE, Neto AG, et al. Textiloma (gossypiboma) mimicking recurrent intracranial tumor. Arch Pathol Lab Med 2004;128:749-58

5. Kothbauer KF, Jallo GI, Siffert J, et al. Foreign body reaction to hemostatic materials mimicking recurrent brain tumor. J Neurosurg 2001;95:503-06

6. Brochert A, Reynolds T, Baker R. MRI in a case of muslin-induced granuloma. Neuroradiology 2003;45:82-84

7. Nishio Y, Hayashi N, Hamada H, et al. A case of delayed brain abscess due to a retained intracranial wooden foreign body: a case report and review of the last 20 years. Acta Neurochir 2004;146:847-50

8. Gibbs VC, Coakley FD, Reines HD. Preventable errors in the operating room retained foreign bodies after surgery - part I. Curr Probl Surg 2007;44:281-337 